\title{
HARMONIC MORPHISMS FROM HOMOGENEOUS HADAMARD MANIFOLDS
}

\author{
SIGMUNDUR GUDMUNDSSON AND JONAS NORDSTRÖM
}

\begin{abstract}
We present a new method for manufacturing complex-valued harmonic morphisms from a wide class of Riemannian Lie groups. This yields new solutions from an important family of homogeneous Hadamard manifolds. We also give a new method for constructing left-invariant foliations on a large class of Lie groups producing harmonic morphisms.
\end{abstract}

\section{INTRODUCTION}

The notion of a minimal submanifold of a given ambient space is of great importance in differential geometry. Harmonic morphisms $\phi:(M, g) \rightarrow$ $(N, h)$ between Riemannian manifolds are useful tools for the construction of such objects. They are solutions to over-determined non-linear systems of partial differential equations determined by the geometric data of the manifolds involved. For this reason harmonic morphisms are difficult to find and have no general existence theory, not even locally. On the contrary there exist 3-dimensional Lie groups not admitting any solutions, independent of which left-invariant Riemannian metrics they are equipped with, see [9] and [10]. This makes the existence theory particularly interesting.

If the codomain is a surface the problem is invariant under conformal changes of the metric on $N^{2}$. Therefore, at least for local studies, the codomain can be taken to be the complex plane with its standard flat metric. Complex-valued harmonic morphism $\phi:(M, g) \rightarrow \mathbb{C}$ from Riemannian manifolds generalize holomorphic functions $f:(M, g, J) \rightarrow \mathbb{C}$ from Kähler manifolds and posess many of their highly interesting properties. The theory of harmonic morphisms can therefore be seen as a generalization of complex analysis.

In this paper we are interested in the existence of complex-valued harmonic morphisms from Lie groups equipped with left-invariant Riemannian metrics. We give a new method for manufacturing solutions on a large family of such spaces. Our following main result is a wide-going generalization of the recent Theorem 12.1 of [9].

Theorem 1.1. Let $G=N \rtimes A$ be a semi-direct product of the connected and simply connected Lie groups $A$ and $N$. Let $G$ be equipped with a leftinvariant metric $g$ and $\mathfrak{g}=\mathfrak{a} \oplus \mathfrak{k} \oplus \mathfrak{m}$ be an orthogonal decomposition of the

2000 Mathematics Subject Classification. 58E20, 53C43, 53C12.

Key words and phrases. harmonic morphisms, minimal submanifolds, Lie groups. 
Lie algebra $\mathfrak{g}$ of $G$ such that $\mathfrak{a}$ and $\mathfrak{n}=\mathfrak{k} \oplus \mathfrak{m}$ are the Lie algebras of $A$ and $N$, respectively. Let $K$ be a closed simply connected subgroup of $N$ with Lie algebra $\mathfrak{k}$ such that

(i) $[\mathfrak{a}, \mathfrak{k}] \subset \mathfrak{k}$,

(ii) $[\mathfrak{a}, \mathfrak{m}] \subset \mathfrak{m}$,

(iii) $[\mathfrak{k} \oplus \mathfrak{m}, \mathfrak{k} \oplus \mathfrak{m}] \subset \mathfrak{k}$,

(iv) trace $\operatorname{ad}_{Z}=0$ for all $Z \in \mathfrak{m}$,

(v) there exists a $\lambda \in \mathfrak{a}^{*}$ such that for each $H \in \mathfrak{a}$ and $Z \in \mathfrak{m}$

$$
\left(\operatorname{ad}_{H}+\operatorname{ad}_{H}^{t}\right)(Z)=2 \lambda(H) \cdot Z .
$$

Then there exists a harmonic morphism $\Phi: G \rightarrow \mathbb{R}^{m}$, where $m=\operatorname{dim} \mathfrak{m}$.

We use our method to produce many new examples of complex-valued harmonic morphisms from Lie groups. We show how the construction can be applied to an important class of homogeneous Hadamard manifolds including the well-known Carnot spaces, see Theorem 10.3. We conclude this paper with a new recipe for constructing conformal foliations on Lie groups producing harmonic morphisms, see Theorem 12.1 .

For the general theory of harmonic morphisms, we refer to the exhaustive book [3] and the on-line bibliography [6] of papers.

\section{HARMONIC MORPHISMS}

Let $M$ and $N$ be two manifolds of dimensions $m$ and $n$, respectively. A Riemannian metric $g$ on $M$ gives rise to the notion of a Laplacian on $(M, g)$ and real-valued harmonic functions $f:(M, g) \rightarrow \mathbb{R}$. This can be generalized to the concept of harmonic maps $\phi:(M, g) \rightarrow(N, h)$ between Riemannian manifolds, which are solutions to a semi-linear system of partial differential equations, see 3 .

Definition 2.1. A map $\phi:(M, g) \rightarrow(N, h)$ between Riemannian manifolds is called a harmonic morphism if, for any harmonic function $f: U \rightarrow \mathbb{R}$ defined on an open subset $U$ of $N$ with $\phi^{-1}(U)$ non-empty, $f \circ \phi: \phi^{-1}(U) \rightarrow$ $\mathbb{R}$ is a harmonic function.

The following characterization of harmonic morphisms between Riemannian manifolds is due to Fuglede and Ishihara. For the definition of horizontal (weak) conformality we refer to [3].

Theorem 2.2. [5, 12] A map $\phi:(M, g) \rightarrow(N, h)$ between Riemannian manifolds is a harmonic morphism if and only if it is a horizontally (weakly) conformal harmonic map.

The next result of Baird and Eells gives the theory of harmonic morphisms a strong geometric flavour and shows that the case when $n=2$ is particularly interesting. The conditions characterizing harmonic morphisms are then independent of conformal changes of the metric on the surface $N^{2}$. 
Theorem 2.3. 2] Let $\phi:\left(M^{m}, g\right) \rightarrow\left(N^{n}, h\right)$ be a horizontally (weakly) conformal map between Riemannian manifolds. If

(i) $n=2$, then $\phi$ is harmonic if and only if $\phi$ has minimal fibres at regular points;

(ii) $n \neq 2$, then two of the following conditions imply the other:

(a) $\phi$ is a harmonic map,

(b) $\phi$ has minimal fibres at regular points,

(c) $\phi$ is horizontally homothetic.

We are interested in complex-valued functions $\phi, \psi:(M, g) \rightarrow \mathbb{C}$ from Riemannian manifolds. In that situation the metric $g$ induces the complexvalued Laplacian $\tau(\phi)$ and the gradient $\operatorname{grad}(\phi)$ with values in the complexified tangent bundle $T^{\mathbb{C}} M$ of $M$. We extend the metric $g$ to be complex bilinear on $T^{\mathbb{C}} M$ and define the symmetric bilinear operator $\kappa$ by

$$
\kappa(\phi, \psi)=g(\operatorname{grad}(\phi), \operatorname{grad}(\psi)) .
$$

Two maps $\phi, \psi: M \rightarrow \mathbb{C}$ are said to be orthogonal if

$$
\kappa(\phi, \psi)=0 \text {. }
$$

The harmonicity and horizontal conformality of $\phi:(M, g) \rightarrow \mathbb{C}$ are expressed by the relations

$$
\tau(\phi)=0 \text { and } \kappa(\phi, \phi)=0 .
$$

Definition 2.4. [7] Let $(M, g)$ be a Riemannian manifold. Then a set

$$
\Omega=\left\{\phi_{k}: M \rightarrow \mathbb{C} \mid k \in I\right\}
$$

of complex-valued functions is said to be an orthogonal harmonic family on $M$ if, for all $\phi, \psi \in \Omega$,

$$
\tau(\phi)=0 \text { and } \kappa(\phi, \psi)=0 .
$$

The next result shows that orthogonal harmonic families can be useful for producing a variety of harmonic morphisms.

Theorem 2.5. [8] Let $(M, g)$ be a Riemannian manifold and

$$
\Omega=\left\{\phi_{k}: M \rightarrow \mathbb{C} \mid k=1, \ldots, n\right\}
$$

be a finite orthogonal harmonic family on $(M, g)$. Let $\Phi: M \rightarrow \mathbb{C}^{n}$ be the map given by $\Phi=\left(\phi_{1}, \ldots, \phi_{n}\right)$ and $U$ be an open subset of $\mathbb{C}^{n}$ containing the image $\Phi(M)$ of $\Phi$. If $\mathcal{H}=\left\{h_{i}: U \rightarrow \mathbb{C} \mid i \in \mathbb{C}\right\}$ is a family of holomorphic functions then the family $\mathcal{F}$ given by

$$
\mathcal{F}=\left\{\psi: M \rightarrow \mathbb{C} \mid \psi=h\left(\phi_{1}, \ldots, \phi_{n}\right), h \in \mathcal{H}\right\}
$$

is an orthogonal harmonic family on $M$. In particular, every element of $\mathcal{F}$ is a harmonic morphism.

The problem of finding an orthogonal harmonic family on a Riemannian manifold can often be reduced to finding a harmonic morphism with values in $\mathbb{R}^{n}$. 
Proposition 2.6. 9] Let $\Phi:(M, g) \rightarrow \mathbb{R}^{n}$ be a harmonic morphism from a Riemannian manifold to the standard Euclidean $\mathbb{R}^{n}$ with $n \geq 2$. If $V$ is an isotropic subspace of $\mathbb{C}^{n}$ then

$$
\Omega_{V}=\left\{\phi_{v}(x)=(\Phi(x), v) \mid v \in V\right\}
$$

is an orthogonal harmonic family of complex-valued functions on $(M, g)$.

Here $(\cdot, \cdot)$ refers to the standard symmetric bilinear form on the complex linear space $\mathbb{C}^{n}$.

\section{HARMONIC MORPHISMS FROM LIE GROUPS}

In this section we introduce a new method for manufacturing complexvalued harmonic morphisms from a large class of Lie groups. This is a wide-going generalization of a construction recently presented in [9].

Theorem 3.1. Let $G=N \rtimes A$ be a semi-direct product of the connected and simply connected Lie groups $A$ and $N$. Let $G$ be equipped with a leftinvariant metric $g$ and $\mathfrak{g}=\mathfrak{a} \oplus \mathfrak{k} \oplus \mathfrak{m}$ be an orthogonal decomposition of the Lie algebra $\mathfrak{g}$ of $G$ such that $\mathfrak{a}$ and $\mathfrak{n}=\mathfrak{k} \oplus \mathfrak{m}$ are the Lie algebras of $A$ and $N$, respectively. Let $K$ be a closed simply connected subgroup of $N$ with Lie algebra $\mathfrak{k}$ such that

(i) $[\mathfrak{a}, \mathfrak{k}] \subset \mathfrak{k}$,

(ii) $[\mathfrak{a}, \mathfrak{m}] \subset \mathfrak{m}$,

(iii) $[\mathfrak{k} \oplus \mathfrak{m}, \mathfrak{k} \oplus \mathfrak{m}] \subset \mathfrak{k}$,

(iv) trace $\operatorname{ad}_{Z}=0$ for all $Z \in \mathfrak{m}$,

(v) there exists $a \lambda \in \mathfrak{a}^{*}$ such that for each $H \in \mathfrak{a}$ and $Z \in \mathfrak{m}$

$$
\left(\operatorname{ad}_{H}+\operatorname{ad}_{H}^{t}\right)(Z)=2 \lambda(H) \cdot Z \text {. }
$$

Then there exists a harmonic morphism $\Phi: G \rightarrow \mathbb{R}^{m}$, where $m=\operatorname{dim} \mathfrak{m}$.

The following proof of Theorem 3.1 is an amended version of the proof of the special case presented in Theorem 12.1 of [9].

Proof. The subalgebra $\mathfrak{k}$ is an ideal of both $\mathfrak{g}$ and $\mathfrak{n}$, so $K$ is a normal subgroup of $G$ and $N$. The group $N / K$ is connected and Abelian since $[\mathfrak{n}, \mathfrak{n}] \subset \mathfrak{k}$. Both $N$ and $K$ are simply connected so $N / K$ is simply connected and hence isomorphic to $\mathbb{R}^{m}$.

Equip $N$ with the metric induced by $g$ on $G$ and suppose that $N / K$ has the unique left-invariant metric $h$ such that the homogeneous projection

$$
\pi:(N, g) \rightarrow(N / K, h)
$$

with $\pi(n)=n K$ is a Riemannian submersion. Define the map $\Psi: G \rightarrow N$ by $\Psi: n a \mapsto n$ and let $\Phi: G \rightarrow N / K$ be the composition $\Phi=\pi \circ \Psi$. We will show that $\Phi$ is a harmonic morphism.

For a point $n K \in N / K$ we have $\Phi^{-1}(n K)=n K A=n A K$. The tangent space of $G$ at $e$ is the Lie algebra $\mathfrak{g}$ and the vertical and horizontal spaces 
of $\Phi$ at $e \in G$ are given by

$$
\mathcal{V}_{e}=\mathfrak{a}+\mathfrak{k} \text { and } \mathcal{H}_{e}=\mathfrak{m}
$$

At a generic point $n a \in G$ the vertical and horizontal spaces of $\Phi$ are given by the left translate by $d L_{n a}$ i.e.

$$
\begin{aligned}
\mathcal{V}_{n a}=\left(d L_{n a}\right)_{e}(\mathfrak{a} & +\mathfrak{k}) \text { and } \mathcal{H}_{n a}=\left(d L_{n a}\right)_{e}(\mathfrak{m}) . \\
d \Phi_{n a}\left(\left(d L_{n a}\right)_{e}(Z)\right) & =\frac{d}{d t}\left[\Phi\left(L_{n a}(\exp (t Z))\right)\right]_{t=0} \\
& =\frac{d}{d t}[\Phi(n \exp (t \sigma(a)(Z) a))]_{t=0} \\
& =\frac{d}{d t}[(n K)(\exp (t \sigma(a))(Z) K)]_{t=0} \\
& =\left(d L_{n K}\right)_{e K}(\sigma(a)(Z)),
\end{aligned}
$$

where $\sigma: A \rightarrow \operatorname{Aut}(\mathfrak{m})$ is given by $\sigma(a)(Z)=\operatorname{Ad}_{a}(Z)$. This implies that if

$$
\rho=d \sigma: \mathfrak{a} \rightarrow \operatorname{End}(\mathfrak{m})
$$

then $\rho(H)$ acts on $\mathfrak{m}$ by the adjoint representation. According to condition (v) this is given by

$$
\rho(H)(Z)=[H, Z]=\lambda(H) Z+\frac{1}{2}\left(\operatorname{ad}_{H}-\operatorname{ad}_{H}^{t}\right) Z .
$$

The map $\rho$ takes values in $\mathbb{R} \cdot \mathrm{I} \oplus \mathfrak{s o}(\mathfrak{m})$ which is the Lie algebra of the conformal group on $\mathfrak{m}$. Hence for each $a \in A$ the map $\sigma(a): \mathfrak{m} \rightarrow \mathfrak{m}$ is conformal. This implies that for $Z, W \in \mathfrak{m}$ we get

$$
\begin{aligned}
& h_{n K}\left(\left(d L_{n K}\right)_{e K}(\sigma(a)(Z)),\left(d L_{n K}\right)_{e K}(\sigma(a)(W))\right) \\
= & h_{e K}(\sigma(a)(Z), \sigma(a)(W)) \\
= & \mu(a)^{2} h_{e K}(Z, W),
\end{aligned}
$$

where $\mu: A \rightarrow \mathbb{R}^{+}$is the dilation of $\sigma(a)$. This is clearly constant along horizontal curves with respect to $\Phi$. This means that $\Phi$ is horizontally homothetic so for proving that $\Phi$ is a harmonic morphism it is sufficient to show that it has minimal fibres.

Since $\Phi^{-1}(n K)=n K A$ and $L_{n}$ is an isometry on $G$ it is enough to show that the fibre $\Phi^{-1}(e K)$ is minimal. Let $\left\{H_{j}\right\}$ be an orthonormal basis for $\mathfrak{a}$ and $\left\{X_{i}\right\}$ an orthonormal basis for $\mathfrak{k}$. Let $\mu^{\mathcal{V}}$ be the mean curvature vector field along $\Phi^{-1}(e K)$. For a generic element $Z \in \mathfrak{m}$ we have $\left[H_{j}, Z\right] \in \mathfrak{m}$ and then conditions (i), (iii) and (v) give

$$
\begin{aligned}
\left\langle\mu^{\mathcal{V}}, Z\right\rangle & =\sum_{j}\left\langle\nabla_{H_{j}} H_{j}, Z\right\rangle+\sum_{i}\left\langle\nabla_{X_{i}} X_{i}, Z\right\rangle \\
& =\sum_{j}\left\langle\left[Z, H_{j}\right], H_{j}\right\rangle+\sum_{i}\left\langle\left[Z, X_{i}\right], X_{i}\right\rangle \\
& =\operatorname{trace} \operatorname{ad}_{Z} \\
& =0 .
\end{aligned}
$$


These calculations show that the fibres are minimal and hence $\Phi$ is a harmonic morphism.

In order to explain condition (v) in Theorem 3.1 we state the following result.

Lemma 3.2. Let $V$ be a real vector space equipped with a Euclidean scalar product $\langle\cdot, \cdot\rangle$. Let $L: V \rightarrow V$ be a linear operator on $V$. Then the following conditions are equivalent.

(i) there exist a $\lambda \in \mathbb{R}$ such that $L=\lambda \cdot I+\left(L-L^{t}\right) / 2$,

(ii) if $Z, W \in V$ such that $|Z|=|W|$ then $\langle L Z, Z\rangle=\langle L W, W\rangle$,

(iii) if $Z, W \in V$ such that $|Z|=|W|=1$ and $\langle Z, W\rangle=0$ then

$$
\langle L Z, Z\rangle-\langle L W, W\rangle=0 \text { and }\langle L Z, W\rangle-\langle L W, Z\rangle=0 .
$$

Proof. The proof is an easy exercise left to the reader.

Remark 3.3. If the Lie algebra $\mathfrak{k}$ is semi-simple then $[\mathfrak{k}, \mathfrak{k}]=\mathfrak{k}$. This means that independent of how we pick $\mathfrak{a}$ and $\mathfrak{m}$ satisfying the conditions in Theorem 3.1 the resulting Lie algebra $\mathfrak{g}=\mathfrak{a} \oplus \mathfrak{k} \oplus \mathfrak{m}$ will not be solvable. This gives a wide class of Lie algebras not covered by Theorem 12.1 of $[9]$.

\section{The CASE OF $\operatorname{dim}(\mathfrak{a}, \mathfrak{k}, \mathfrak{m})=(2,0,2)$}

Let $\mathfrak{g}$ be a 4-dimensional Lie algebra equipped with a Euclidean metric and $\mathfrak{g}=\mathfrak{a} \oplus \mathfrak{k} \oplus \mathfrak{m}$ be an orthogonal decomposition of $\mathfrak{g}$ satisfying the conditions of Theorem 3.1. If $\operatorname{dim}(\mathfrak{a}, \mathfrak{k}, \mathfrak{m})=(2,0,2)$ then there exists an orthonormal basis $\{A, B, Z, W\}$ for $\mathfrak{g}$ such that $A, B \in \mathfrak{a}, Z, W \in \mathfrak{m}$ and

$$
\begin{gathered}
{[A, B]=a A+b B,} \\
{[A, Z]=\alpha Z+\beta W, \quad[A, W]=-\beta Z+\alpha W,} \\
{[B, Z]=x Z+y W, \quad[B, W]=-y Z+x W,}
\end{gathered}
$$

The Jacobi identity gives the following conditions on the real coefficients

$$
a \alpha+b x=0=a \beta+b y,
$$

The Lie algebra $\mathfrak{g}$ is solvable, the vertical foliation generated by $\mathfrak{a}$ is totally geodesic and the horizontal distribution $\mathfrak{m}$ is integrable.

$$
\text { 5. The CASE OF } \operatorname{dim}(\mathfrak{a}, \mathfrak{k}, \mathfrak{m})=(1,1,2)
$$

Let $\mathfrak{g}$ be a 4-dimensional Lie algebra equipped with a Euclidean metric and $\mathfrak{g}=\mathfrak{a} \oplus \mathfrak{k} \oplus \mathfrak{m}$ be an orthogonal decomposition of $\mathfrak{g}$ satisfying the conditions of Theorem 3.1. If $\operatorname{dim}(\mathfrak{a}, \mathfrak{k}, \mathfrak{m})=(1,1,2)$ then there exists an orthonormal basis $\{A, X, Z, W\}$ for $\mathfrak{g}$ such that $A \in \mathfrak{a}, X \in \mathfrak{k}, Z, W \in \mathfrak{m}$ and

$$
\begin{gathered}
{[A, X]=\lambda X,} \\
{[A, Z]=\alpha Z+\beta W, \quad[A, W]=-\beta Z+\alpha W,} \\
{[Z, W]=\theta X .}
\end{gathered}
$$


The Jacobi identity gives the following condition on the real coefficients

$$
\theta(\lambda-2 \alpha)=0
$$

The Lie algebra $\mathfrak{g}$ is solvable and the horizontal distribution $\mathfrak{m}$ is integrable if and only if $\theta=0$.

$$
\text { 6. The CASE OF } \operatorname{dim}(\mathfrak{a}, \mathfrak{k}, \mathfrak{m})=(0,2,2)
$$

Let $\mathfrak{g}$ be a 4-dimensional Lie algebra equipped with a Euclidean metric and $\mathfrak{g}=\mathfrak{a} \oplus \mathfrak{k} \oplus \mathfrak{m}$ be an orthogonal decomposition of $\mathfrak{g}$ satisfying the conditions of Theorem 3.1. If $\operatorname{dim}(\mathfrak{a}, \mathfrak{k}, \mathfrak{m})=(0,2,2)$ then there exists an orthonormal basis $\{X, Y, Z, W\}$ for $\mathfrak{g}$ such that $X, Y \in \mathfrak{k}, Z, W \in \mathfrak{k}$ and

$$
\begin{gathered}
{[X, Y]=z X+w Y,} \\
{[Z, X]=r X+s Y, \quad[Z, Y]=t X-r Y,} \\
{[W, X]=\rho X+\sigma Y, \quad[W, Y]=\tau X-\rho Y,} \\
{[Z, W]=\theta X .}
\end{gathered}
$$

The Jacobi identity gives 8 quadratic equations which can be solved in order to construct a variety of solutions. The horizontal distribution $\mathfrak{m}$ is integrable if and only if $\theta=0$. The fibres are totally geodesic if and only if $r=s+t=\rho=\sigma+\tau=0$. We present our examples by simply listing the non-vanishing Lie brackets in each case.

\section{Example 6.1.}

$$
\begin{gathered}
{[X, Y]=z X+w Y,} \\
{[Z, X]=-\frac{w t}{z} X-\frac{w^{2} t}{z^{2}} Y, \quad[Z, Y]=t X+\frac{w t}{z} Y .} \\
{[W, X]=-\frac{w \tau}{z} X-\frac{w^{2} \tau}{z^{2}} Y, \quad[W, Y]=\tau X+\frac{w \tau}{z} Y .}
\end{gathered}
$$

\section{Example 6.2.}

$$
\begin{gathered}
{[X, Y]=w Y} \\
{[Z, X]=s Y, \quad[W, X]=\sigma Y}
\end{gathered}
$$

Example 6.3.

$$
\begin{gathered}
{[Z, X]=r X+s Y, \quad[Z, Y]=t X-r Y,} \\
{[W, X]=\rho X+\frac{s \rho}{r} Y, \quad[W, Y]=\frac{t \rho}{r} X-\rho Y,} \\
{[Z, W]=\theta X .}
\end{gathered}
$$

\section{Example 6.4.}

$$
\begin{gathered}
{[Z, X]=s Y, \quad[Z, Y]=t X,} \\
{[W, X]=\sigma Y, \quad[W, Y]=\frac{t \sigma}{s} X,} \\
{[Z, W]=\theta X .} \\
7
\end{gathered}
$$




\section{Example 6.5.}

$$
\begin{gathered}
{[W, X]=\rho X+\sigma Y, \quad[W, Y]=\tau X-\rho Y,} \\
{[Z, W]=\theta X .}
\end{gathered}
$$

\section{Example 6.6.}

$$
\begin{gathered}
{[Z, Y]=t X, \quad[W, Y]=\tau X} \\
{[Z, W]=\theta X .}
\end{gathered}
$$

7. The CASe of $\operatorname{dim}(\mathfrak{a}, \mathfrak{k}, \mathfrak{m})=(2,1,2)$

Let $\mathfrak{g}$ be a 5 -dimensional Lie algebra equipped with a Euclidean metric and $\mathfrak{g}=\mathfrak{a} \oplus \mathfrak{k} \oplus \mathfrak{m}$ be an orthogonal decomposition of $\mathfrak{g}$ satisfying the conditions of Theorem 3.1. If $\operatorname{dim}(\mathfrak{a}, \mathfrak{k}, \mathfrak{m})=(2,1,2)$ then there exists an orthonormal basis $\{A, B, X, Z, W\}$ for $\mathfrak{g}$ such that $A, B \in \mathfrak{a}, X \in \mathfrak{k}, Z, W \in \mathfrak{m}$ and

$$
\begin{gathered}
{[A, B]=a A+b B,} \\
{[A, X]=\lambda X, \quad[B, X]=\mu X,} \\
{[A, Z]=\alpha Z+\beta W, \quad[A, W]=-\beta Z+\alpha W,} \\
{[B, Z]=x Z+y W, \quad[B, W]=-y Z+x W,} \\
{[Z, W]=\theta X .}
\end{gathered}
$$

The Jacobi identity gives 5 quadratic conditions for the real coefficients.

$$
\begin{gathered}
\theta(\lambda-2 \alpha)=0=\theta(\mu-2 x), \\
a \alpha+b x=0=a \beta+b y, \\
a \lambda+b \mu=0 .
\end{gathered}
$$

By solving these equations, we get the following examples.

\section{Example 7.1.}

$$
\begin{gathered}
{[A, B]=a A+b B} \\
{[A, X]=-\frac{b \mu}{a} X, \quad[B, X]=\mu X,} \\
{[A, Z]=-\frac{b x}{a} Z-\frac{b y}{a} W, \quad[A, W]=\frac{b y}{a} Z-\frac{b x}{a} W} \\
{[B, Z]=x Z+y W, \quad[B, W]=-y Z+x W .}
\end{gathered}
$$

\section{Example 7.2.}

$$
\begin{gathered}
{[A, B]=a A+b B} \\
{[A, X]=-2 \frac{b x}{a} X, \quad[B, X]=2 x X,} \\
{[A, Z]=-\frac{b x}{a} Z-\frac{b y}{a} W, \quad[A, W]=\frac{b y}{a} Z-\frac{b x}{a} W} \\
{[B, Z]=x Z+y W, \quad[B, W]=-y Z+x W} \\
{[Z, W]=\theta X .} \\
8
\end{gathered}
$$




\section{Example 7.3.}

$$
\begin{gathered}
{[A, X]=2 \alpha X, \quad[B, X]=2 x X} \\
{[A, Z]=\alpha Z+\beta W, \quad[A, W]=-\beta Z+\alpha W} \\
{[B, Z]=x Z+y W, \quad[B, W]=-y Z+x W} \\
{[Z, W]=\theta X .}
\end{gathered}
$$

\section{Example 7.4.}

$$
\begin{gathered}
{[A, B]=b B} \\
{[A, X]=2 \alpha X} \\
{[A, Z]=\alpha Z+\beta W, \quad[A, W]=-\beta Z+\alpha W} \\
{[Z, W]=\theta X .}
\end{gathered}
$$

\section{Example 7.5.}

$$
\begin{gathered}
{[A, X]=\lambda X, \quad[B, X]=\mu X} \\
{[A, Z]=\alpha Z+\beta W, \quad[A, W]=-\beta Z+\alpha W,} \\
{[B, Z]=x Z+y W, \quad[B, W]=-y Z+x W .}
\end{gathered}
$$

\section{Example 7.6.}

$$
\begin{gathered}
{[A, B]=b B,} \\
{[A, X]=\lambda X,} \\
{[A, Z]=\alpha Z+\beta W, \quad[A, W]=-\beta Z+\alpha W,}
\end{gathered}
$$

8. The CASE OF $\operatorname{dim}(\mathfrak{a}, \mathfrak{k}, \mathfrak{m})=(1,2,2)$

Let $\mathfrak{g}$ be a 5 -dimensional Lie algebra equipped with a Euclidean metric and $\mathfrak{g}=\mathfrak{a} \oplus \mathfrak{k} \oplus \mathfrak{m}$ be an orthogonal decomposition of $\mathfrak{g}$ satisfying the conditions of Theorem 3.1. If $\operatorname{dim}(\mathfrak{a}, \mathfrak{k}, \mathfrak{m})=(1,2,2)$ then there exists an orthonormal basis $\{A, X, Y, Z, W\}$ for $\mathfrak{g}$ such that $A \in \mathfrak{a}, X, Y \in \mathfrak{k}$ and $Z, W \in \mathfrak{m}$ and

$$
\begin{gathered}
{[A, X]=\gamma X+\delta Y, \quad[A, Y]=c X+d Y,} \\
{[A, Z]=\alpha Z+\beta W, \quad[A, W]=-\beta Z+\alpha W,} \\
{[X, Y]=z X+w Y,} \\
{[Z, X]=r X+s Y, \quad[Z, Y]=t X-r Y,} \\
{[W, X]=\rho X+\sigma Y, \quad[W, Y]=\tau X-\rho Y,} \\
{[Z, W]=\theta X .}
\end{gathered}
$$

The Jacobi identity gives 20 quadratic equations which can be solved in order to construct a large variety of solutions. In this section we focus our attention on cases with non-integrable horizontal distribution $(\theta \neq 0)$ and non-totally geodesic fibres $(r \neq 0$ or $s+t \neq 0)$.

\section{Example 8.1.}

$$
\begin{gathered}
{[A, Y]=d Y} \\
{[Z, X]=r X, \quad[Z, Y]=-r Y} \\
{[W, X]=\rho X, \quad[W, Y]=-\rho Y,} \\
{[Z, W]=\theta X .}
\end{gathered}
$$




\section{Example 8.2.}

$$
\begin{gathered}
{[A, X]=2 \alpha X, \quad[A, Y]=\alpha X+3 \alpha Y} \\
{[A, Z]=\alpha Z, \quad[A, W]=\alpha W} \\
{[Z, X]=r X+r Y, \quad[Z, Y]=-r X-r Y} \\
{[W, X]=\rho X+\rho Y, \quad[W, Y]=-\rho X-\rho Y} \\
{[Z, W]=\theta X}
\end{gathered}
$$

Example 8.3.

$$
\begin{gathered}
{[A, X]=2 \alpha X, \quad[A, Y]=c X+\alpha Y,} \\
{[A, Z]=\alpha Z, \quad[A, W]=\alpha W,} \\
{[Z, Y]=t X, \quad[W, Y]=\tau X,} \\
{[Z, W]=\theta X .}
\end{gathered}
$$

Example 8.4.

$$
\begin{gathered}
{[A, X]=2 \alpha X, \quad[A, Y]=3 \alpha Y,} \\
{[A, Z]=\alpha Z, \quad[A, W]=\alpha W} \\
{[Z, X]=s Y} \\
{[W, X]=\sigma Y} \\
{[Z, W]=\theta X}
\end{gathered}
$$

\section{Example 8.5.}

$$
\begin{gathered}
{[A, X]=2 \alpha X, \quad[A, Y]=\frac{\alpha r}{s} X+3 \alpha Y,} \\
{[A, Z]=\alpha Z, \quad[A, W]=\alpha W,} \\
{[Z, X]=r X+s Y, \quad[Z, Y]=-\frac{r^{2}}{s} X-r Y,} \\
{[Z, W]=\theta X .}
\end{gathered}
$$

9. The CASE OF $\operatorname{dim}(\mathfrak{a}, \mathfrak{k}, \mathfrak{m})=(1, n, 2)$

Example 9.1. Let $\mathfrak{g}$ be an $(n+3)$-dimensional Lie algebra equipped with a Euclidean metric and $\mathfrak{g}=\mathfrak{a} \oplus \mathfrak{k} \oplus \mathfrak{m}$ be an orthogonal decomposition of $\mathfrak{g}$. Let $\left\{A, X_{1}, \ldots, X_{n}, Z, W\right\}$ be an orthonormal basis for $\mathfrak{g}$ such that $A \in \mathfrak{a}$, $X_{1}, \ldots, X_{n} \in \mathfrak{k}, Z, W \in \mathfrak{m}$ and

$$
\begin{aligned}
{\left[A, X_{k}\right] } & =c_{k 1} X_{1}+\cdots+c_{k n} X_{n} \\
{[A, Z] } & =\alpha Z+\beta W \\
{[A, W] } & =-\beta Z+\alpha W .
\end{aligned}
$$

The Jacobi identity is satisfied for any choice of real numbers $\alpha, \beta$ and $c_{k j}$. The Lie algebra $\mathfrak{g}$ is solvable and satisfies the conditions in Theorem 3.1.

If $n \geq 2, c_{k j}=0$ for $k<j$ and for some $r<s$ we have $c_{r r}=c_{s s}$ and $c_{s r} \neq 0$ or if $\beta \neq 0$, then $\operatorname{ad}_{A}$ is not diagonalizable and we get new examples of harmonic morphisms not covered by Theorem 12.1 of 9 . 
Example 9.2. Let $\mathfrak{g}$ be an $(n+3)$-dimensional Lie algebra equipped with a Euclidean metric and $\mathfrak{g}=\mathfrak{a} \oplus \mathfrak{k} \oplus \mathfrak{m}$ be an orthogonal decomposition of $\mathfrak{g}$. Let $\left\{A, X_{1}, \ldots, X_{n}, Z, W\right\}$ be an orthonormal basis for $\mathfrak{g}$ such that $A \in \mathfrak{a}$, $X_{1}, \ldots, X_{n} \in \mathfrak{k}$ and $Z, W \in \mathfrak{m}$ and

$$
\begin{aligned}
{\left[A, X_{k}\right] } & =X_{k} \\
{[A, Z] } & =\alpha Z+\beta W \\
{[A, W] } & =-\beta Z+\alpha W \\
{[Z, W] } & =X_{1} .
\end{aligned}
$$

Then the Jacobi identity implies that $\alpha=1 / 2$. The Lie algebra $\mathfrak{g}$ is solvable and satisfies the conditions in Theorem 3.1. The horizontal distribution $\mathfrak{m}$ is not integrable.

\section{HomogeneOus HadAmard MANifolds}

Homogeneous Hadamard manifolds are simply connected Riemannian homogeneous spaces with non-positive sectional curvature. Every such manifold $\left(M^{m}, g\right)$ is diffeomorphic to the standard $\mathbb{R}^{m}$ and it is actually isometric to a solvable Riemannian Lie group, see [15], [1] and [1]. Important examples are the irreducible Riemannian symmetric spaces of non-compact type and the Damek-Ricci spaces, see [4].

If $S$ is a Riemannian Lie group with Lie algebra $\mathfrak{s}$, of non-positive curvature, then there exists an orthogonal decomposition $\mathfrak{s}=\mathfrak{a} \oplus \mathfrak{n}$ of $\mathfrak{s}$, where $\mathfrak{n}=[\mathfrak{s}, \mathfrak{s}]$. The subalgebra $\mathfrak{n}$ is nilpotent and $\mathfrak{a}$ is Abelian. It follows from the Jacobi identity that if $H_{1}, H_{2} \in \mathfrak{a}$ and $X \in \mathfrak{n}$ then

$$
\left(\operatorname{ad}_{H_{1}} \circ \operatorname{ad}_{H_{2}}-\operatorname{ad}_{H_{2}} \circ \operatorname{ad}_{H_{1}}\right)(X)=0
$$

i.e. the adjoint action of $\mathfrak{a}$ on $\mathfrak{n}$ is Abelian. This implies that the complexification $\mathfrak{n}^{\mathbb{C}}$ of $\mathfrak{n}$ is a direct sum

$$
\mathfrak{n}^{\mathbb{C}}=\bigoplus_{\lambda} \mathfrak{n}_{\lambda}^{\mathbb{C}}
$$

of the non-trivial root spaces

$$
\mathfrak{n}_{\lambda}^{\mathbb{C}}=\left\{X \in \mathfrak{n}^{\mathbb{C}} \mid\left(\operatorname{ad}_{H}-\lambda(H)\right)^{k} X=0 \text { for some } k>0 \text { and all } H \in \mathfrak{a}\right\},
$$

where $\lambda \in\left(\mathfrak{a}^{\mathbb{C}}\right)^{*}$ are the corresponding roots. For a root $\lambda=\alpha \pm i \beta \in\left(\mathfrak{a}^{\mathbb{C}}\right)^{*}$ the generalized root space $\mathfrak{n}_{\alpha, \beta}$ is given by

$$
\mathfrak{n}_{\alpha, \beta}=\mathfrak{n} \cap\left(\mathfrak{n}_{\lambda}^{\mathbb{C}} \oplus \mathfrak{n}_{\bar{\lambda}}^{\mathbb{C}}\right) .
$$

Then the vector space $\mathfrak{n}$ is the direct sum of generalized root spaces

$$
\mathfrak{n}=\bigoplus_{\lambda=\alpha \pm i \beta} \mathfrak{n}_{\alpha, \beta} .
$$


Definition 10.1. Let $V$ be a complex vector space equipped with a Hermitian scalar product and $L: V \rightarrow V$ be a linear operator on $V$. Further define $N(L): V \rightarrow V$ by

$$
4 N(L)=\left(L+L^{*}\right)^{2}+\left[L+L^{*}, L-L^{*}\right] .
$$

Then the operator $L$ is said to be

(i) normal if $L L^{*}=L^{*} L$, and

(ii) almost normal if $N(L)$ is semi-positive definite i.e. for all $Z \in V$

$$
\langle N(L)(Z), Z\rangle \geq 0 \text {. }
$$

For homogeneous Hadamard manifolds, Azencott and Wilson have in [1] shown that the complex linear extension $\left.\operatorname{ad}_{H}\right|_{\mathfrak{n}} \mathbb{C}: \mathfrak{n}^{\mathbb{C}} \rightarrow \mathfrak{n}^{\mathbb{C}}$ of $\left.\operatorname{ad}_{H}\right|_{\mathfrak{n}}$ is almost normal for all $H \in \mathfrak{a}$. It is easily seen that any normal operator $L$ is also almost normal. We shall now focus our attention on the special case of normality.

Lemma 10.2. Let $\mathfrak{n}_{\alpha, \beta}$ be a generalized root space of $\mathfrak{n}$ such that the operator $\left.\operatorname{ad}_{H}\right|_{\mathfrak{n}_{\alpha, \beta}}$ is normal for all $H \in \mathfrak{a}$. Then

$$
\left.\operatorname{ad}_{H}\right|_{\mathfrak{n}_{\alpha, \beta}}=\alpha(H) I_{\mathfrak{n}_{\alpha, \beta}}+\left.\frac{1}{2}\left(\operatorname{ad}_{H}-\operatorname{ad}_{H}^{*}\right)\right|_{\mathfrak{n}_{\alpha, \beta}}
$$

Proof. The complex linear extension

$$
\left.\operatorname{ad}_{H}\right|_{\mathfrak{n}_{\lambda}^{\mathbb{C}} \oplus \mathfrak{n}_{\bar{\lambda}}^{\mathbb{C}}}: \mathfrak{n}_{\lambda}^{\mathbb{C}} \oplus \mathfrak{n}_{\bar{\lambda}}^{\mathbb{C}} \rightarrow \mathfrak{n}_{\lambda}^{\mathbb{C}} \oplus \mathfrak{n}_{\bar{\lambda}}^{\mathbb{C}}
$$

of $\left.\operatorname{ad}_{H}\right|_{\mathfrak{n}_{\alpha, \beta}}$ is normal and hence diagonalizable over $\mathbb{C}$. Let $\left\{X_{1}, \ldots, X_{n}\right\}$ and $\left\{Y_{1}, \ldots, Y_{n}\right\}$ be orthonormal basis of eigenvectors for $\mathfrak{n}_{\lambda}^{\mathbb{C}}$ and $\mathfrak{n}_{\bar{\lambda}}^{\mathbb{C}}$, respectively. For $X \in \mathfrak{n}_{\lambda}$ we then get

$$
\begin{aligned}
\operatorname{ad}_{H}^{*}(X) & =\sum_{k=1}^{n}\left(\left\langle\operatorname{ad}_{H}^{*}(X), X_{k}\right\rangle X_{k}+\left\langle\operatorname{ad}_{H}^{*}(X), Y_{k}\right\rangle Y_{k}\right) \\
& =\sum_{k=1}^{n}\left(\left\langle X, \operatorname{ad}_{H}\left(X_{k}\right)\right\rangle X_{k}+\left\langle X, \operatorname{ad}_{H}\left(Y_{k}\right)\right\rangle Y_{k}\right) \\
& =\sum_{k=1}^{n}\left\langle X, \lambda(H) X_{k}\right\rangle X_{k} \\
& =\sum_{k=1}^{n} \bar{\lambda}(H)\left\langle X, X_{k}\right\rangle X_{k} \\
& =\bar{\lambda}(H) X .
\end{aligned}
$$

Similarily, we see that if $Y \in \mathfrak{n}_{\bar{\lambda}}$ then $\operatorname{ad}_{H}^{*}(Y)=\lambda(H) Y$ and hence

$$
\left(\operatorname{ad}_{H}+\operatorname{ad}_{H}^{*}\right)(Z)=(\lambda(H)+\bar{\lambda}(H)) Z=2 \alpha(H) Z
$$

for all $Z \in \mathfrak{n}_{\lambda} \oplus \mathfrak{n}_{\bar{\lambda}}$. 
We shall now apply our general result of Theorem 3.1 to construct harmonic morphisms from an important class of homogeneous Hadamard manifolds.

Theorem 10.3. Let the solvable Riemannian Lie group $S$ be a homogeneous Hadamard manifold with Lie algebra $\mathfrak{s}=\mathfrak{a}+\mathfrak{n}$. Furthermore assume that there exists a generalized root space $\mathfrak{n}_{\alpha, \beta}$ of $\mathfrak{n}$ of dimension $m \geq 2$ such that

(i) $\left.\operatorname{ad}_{H}\right|_{\mathfrak{n}_{\alpha, \beta}}$ is normal for all $H \in \mathfrak{a}$,

(ii) $[\mathfrak{n}, \mathfrak{n}]$ is contained in the orthogonal complement $\mathfrak{n}_{\alpha, \beta}^{\perp}$ of $\mathfrak{n}_{\alpha, \beta}$ in $\mathfrak{n}$,

(iii) $\operatorname{ad}_{H}\left(\mathfrak{n}_{\alpha, \beta}^{\perp}\right) \subset \mathfrak{n}_{\alpha, \beta}^{\perp}$ for all $H \in \mathfrak{a}$.

Then there exists a harmonic morphism $\Phi: S \rightarrow \mathbb{R}^{m}$.

Proof. In this situation we have an orthogonal decomposition $\mathfrak{s}=\mathfrak{a} \oplus \mathfrak{k} \oplus \mathfrak{m}$ of the Lie algebra $\mathfrak{s}$ where $\mathfrak{m}=\mathfrak{n}_{\alpha, \beta}$ and $\mathfrak{k}=\mathfrak{n}_{\alpha, \beta}^{\perp}$. It is now easy to check, using Lemma 10.2 and the fact that $\mathfrak{n}$ is nilpotent, that the conditions of Theorem 3.1 are satisfied.

Example 10.4. The Carnot spaces form an interesting family of homogeneous Hadamard manifolds, see [13]. Such a space $(S, g)$ is a solvable Riemannian Lie group with Lie algebra $\mathfrak{s}$ and an orthogonal decomposition

$$
\mathfrak{s}=\mathfrak{a} \oplus \bigoplus_{r=1}^{k} \mathfrak{n}_{r}
$$

of $\mathfrak{s}$ such that $\mathfrak{a}$ is a one dimensional subalgebra and the adjoint action of $\mathfrak{a}$ satisfies

$$
\operatorname{ad}_{H}\left(X_{r}\right)=\left[H, X_{r}\right]=r \cdot X_{r},
$$

for all $H \in \mathfrak{a}$ and $X_{r} \in \mathfrak{n}_{r}$. It is an immediate consequence of the Jacobi identity that

$$
\left[\mathfrak{n}_{r}, \mathfrak{n}_{s}\right] \subset \mathfrak{n}_{r+s}
$$

where $\mathfrak{n}_{r+s}=0$ if $k \leq r+s$.

Let us assume that the dimension of $\mathfrak{n}_{1}$ is at least 2 and put $\mathfrak{m}=\mathfrak{n}_{1}$ and $\mathfrak{k}=\mathfrak{n}_{2} \oplus \cdots \oplus \mathfrak{n}_{k}$. Then we have an orthogonal decomposition $\mathfrak{s}=\mathfrak{a} \oplus \mathfrak{k} \oplus \mathfrak{m}$ satisfying the conditions of Theorem 3.1 .

\section{EXAmples}

We have constructed many new examples of complex-valued harmonic morphisms from Riemannian Lie groups. It turns out that several of these spaces are actually homogeneous Hadamard manifolds. This can be checked by calculating their sectional curvatures. For this we have used a Maple programme written by Valentine Svensson, see [14]. Here we list some of these examples and conditions for the real parameters, ensuring non-positive curvature in each case.

Example 7.1: $a^{3}<b^{2} \mu, a^{3}<b^{2} x, b^{2}<a \mu, b^{2}<a x$ and $0<a, \mu, x$. 
Example 7.2: $\theta^{2} a^{2}<8 x^{2}\left(a^{2}+b^{2}\right), a^{3}<b^{2} x, b^{2}<a x$ and $0<a, x$.

Example 7.3: $\theta^{2}<8\left(x^{2}+a^{2}\right)$.

Example 7.4: $0<\alpha b$ and $\theta^{2}<8 \alpha^{2}$.

Example 7.5: $0<\mu x+\lambda \alpha$.

Example 7.6: $0<\alpha b, 0<\alpha \lambda$ and $0<b \lambda$.

Example 8.2: $4\left(\rho^{2}+r^{2}\right)<23 \alpha^{2}, \theta^{2}<8 \alpha^{2}+4 r^{2}$ and $\theta^{2}<8 \alpha^{2}+4 \rho^{2}$.

Example 8.3: $c^{2}<16 \alpha^{2}, \theta^{2}+\tau^{2}<8 \alpha^{2}, \theta^{2}+t^{2}<8 \alpha^{2}$ and $t^{2}+\tau^{2}+c^{2}<8 \alpha^{2}$.

Example 8.4: $s^{2}<12 \alpha^{2}, \sigma^{2}<12 \alpha^{2}, \theta^{2}<3 s^{2}+8 \alpha^{2}$ and $\theta^{2}<3 \sigma^{2}+8 \alpha^{2}$.

Example 8.5: $r^{2}<16 s^{2}, \theta^{2}<8 \alpha^{2}, 2 r^{2} s^{2}+r^{4}+\alpha^{2} r^{2}+s^{4}<24 \alpha^{2} s^{2}$, $\theta^{2} s^{2}+r^{4}<3 s^{4}+2 r^{2} s^{2}+8 \alpha^{2} s^{2}$ and $s^{4}<12 \alpha^{2} s^{2}+2 r^{2} s^{2}+3 r^{4}$.

Example 9.2: no conditions are needed.

\section{Conformal foliations on Lie groups}

In this section we present a new method for constructing left-invariant foliations on a wide class of Lie groups producing harmonic morphisms. For the theory of foliations producing harmonic morphisms we recommend the standard reference [3].

Theorem 12.1. Let $G$ be a Lie group equipped with a left-invariant Riemannian metric. Let $\mathfrak{g}=\mathfrak{a} \oplus \mathfrak{k} \oplus \mathfrak{m}$ be an orthogonal decomposition of the Lie algebra $\mathfrak{g}$ of $G$ such that

(i) $\mathfrak{a} \oplus \mathfrak{k}$ is a subalgebra of $\mathfrak{g}$,

(ii) $[\mathfrak{a}, \mathfrak{m}] \subset \mathfrak{k} \oplus \mathfrak{m}$,

(iii) $[\mathfrak{k} \oplus \mathfrak{m}, \mathfrak{k} \oplus \mathfrak{m}] \subset \mathfrak{k}$.

Furthermore we assume that if $H \in \mathfrak{a}$ and $Z, W \in \mathfrak{m}$ such that $|Z|=|W|=1$ and $\langle Z, W\rangle=0$, then

(iv) $\left\langle\operatorname{ad}_{H} Z, Z\right\rangle-\left\langle\operatorname{ad}_{H} W, W\right\rangle=0$,

(v) $\left\langle\operatorname{ad}_{H} Z, W\right\rangle+\left\langle\operatorname{ad}_{H} W, Z\right\rangle=0$,

(vi) trace $\operatorname{ad}_{Z}=0$.

Under the above conditions the distribution $\mathcal{V}=\mathfrak{a} \oplus \mathfrak{k}$ is integrable and the corresponding foliation produces harmonic morphisms.

Proof. For the integrability of the distribution $\mathcal{V}$ is trivial. The minimality of the leaves of the foliation $\mathcal{V}$, is an immediate consequence of the following 
calculations, implied by conditions (ii), (iii) and (vi).

$$
\begin{aligned}
\operatorname{trace} B^{\mathcal{V}} & =\sum_{k}\left(\sum_{r}\left(\left\langle\left[Z_{k}, A_{r}\right], A_{r}\right\rangle\right)+\sum_{s}\left(\left\langle\left[Z_{k}, X_{s}\right], X_{s}\right\rangle\right)\right) Z_{k} \\
& =\sum_{k}\left(\operatorname{trace} \operatorname{ad}_{Z_{k}}\right) Z_{k} \\
& =0 .
\end{aligned}
$$

For the conformality of the foliation $\mathcal{V}$, we see that conditions (iii), (iv) and (v) obtain

$$
\begin{aligned}
B^{\mathcal{H}}(Z, Z)-B^{\mathcal{H}}(W, W) & =\sum_{r}\left(\left\langle\left[A_{r}, Z\right], Z\right\rangle-\left\langle\left[A_{r}, W\right], W\right\rangle\right) A_{r} \\
& =0
\end{aligned}
$$

and

$$
\begin{aligned}
B^{\mathcal{H}}(Z, W) & =\sum_{r}\left(\left\langle\left[A_{r}, Z\right], Y\right\rangle+\left\langle\left[A_{r}, W\right], X\right\rangle\right) A_{r} \\
& =0 .
\end{aligned}
$$

It follows from Corollary 4.7.7 of [3] that if the dimension of $\mathfrak{m}$ is 2 then we are done. If that is not the case let $\omega$ be the real-valued 1-form on $G$ given by

$$
\omega(E)=\frac{n-2}{n} \sum_{k}\left\langle\mathcal{V} \nabla_{Z_{k}} Z_{k}, E\right\rangle-\sum_{r}\left\langle\mathcal{H} \nabla_{A_{r}} A_{r}, E\right\rangle-\sum_{s}\left\langle\mathcal{H} \nabla_{X_{s}} X_{s}, E\right\rangle .
$$

To prove the statement in this case, it is sufficient to show that $d \omega=0$ i.e. the 1 -form $\omega$ is closed. Let $A \in \mathfrak{a}, X \in \mathfrak{k}$ and $Z \in \mathfrak{m}$ then

$$
\begin{aligned}
\omega(A) & =\frac{n-2}{n} \sum_{k}\left\langle\mathcal{V} \nabla_{Z_{k}} Z_{k}, A\right\rangle \\
& =\frac{n-2}{n} \sum_{k}\left\langle\left[A, Z_{k}\right], Z_{k}\right\rangle .
\end{aligned}
$$

Note that since the vector fields that we have chosen are left-invariant the function $\omega(A)$ is constant. Next we see that

$$
\begin{aligned}
\omega(X) & =\frac{n-2}{n} \sum_{k}\left\langle\mathcal{V} \nabla_{Z_{k}} Z_{k}, X\right\rangle \\
& =\frac{n-2}{n} \sum_{k}\left\langle\left[X, Z_{k}\right], Z_{k}\right\rangle \\
& =0
\end{aligned}
$$

and similarly

$$
\begin{aligned}
\omega(Z) & =-\sum_{r}\left\langle\mathcal{H} \nabla_{A_{r}} A_{r}, Z\right\rangle-\sum_{s}\left\langle\mathcal{H} \nabla_{X_{s}} X_{s}, Z\right\rangle \\
& =-\sum_{r}\left\langle\left[Z, A_{r}\right], A_{r}\right\rangle-\sum_{s}\left\langle\left[Z, X_{s}\right], X_{s}\right\rangle
\end{aligned}
$$




$$
\begin{aligned}
& =- \text { trace } \operatorname{ad}_{Z} \\
& =0 .
\end{aligned}
$$

If $E, F \in \mathfrak{g}$ are left-invariant then the exterior derivative $d \omega$ satisfies

$$
d \omega(E, F)=E(\omega(F))-F(\omega(E))-\omega([E, F])=-\omega([E, F])=0,
$$

since $\omega$ vanishes on $[\mathfrak{g}, \mathfrak{g}] \subset \mathfrak{k} \oplus \mathfrak{m}$.

\section{Acknowledgements}

The authors are grateful to Martin Svensson for very useful discussions on this work.

\section{REFERENCES}

[1] R. Azencott, E. Wilson, Homogeneous Manifolds with Negative Curvature. I, Trans. Amer. Math. Soc. 215 (1976), 323-362.

[2] P. Baird, J. Eells, A conservation law for harmonic maps, Geometry Symposium Utrecht 1980, Lecture Notes in Mathematics 894, 1-25, Springer (1981).

[3] P. Baird, J. C. Wood, Harmonic morphisms between Riemannian manifolds, London Math. Soc. Monogr. 29, Oxford Univ. Press (2003).

[4] J. Berndt, F. Tricerri, L. Vanhecke, Generalized Heisenberg groups and Damek-Ricci harmonic spaces, Lecture Notes in Mathematics 1598, Springer (1995).

[5] B. Fuglede, Harmonic morphisms between Riemannian manifolds, Ann. Inst. Fourier 28 (1978), 107-144.

[6] S. Gudmundsson, The Bibliography of Harmonic Morphisms, http://www.matematik.lu.se/ matematiklu/personal/sigma/harmonic/bibliography.html

[7] S. Gudmundsson, On the existence of harmonic morphisms from symmetric spaces of rank one, Manuscripta Math. 93 (1997), 421-433.

[8] S. Gudmundsson, M. Svensson Harmonic morphisms from the Grassmannians and their non-compact duals, Ann. Global Anal. Geom. 30 (2006), 313-333

[9] S. Gudmundsson, M. Svensson Harmonic morphisms from solvable Lie groups, Math. Proc. Cambridge Philos. Soc. 147 (2009), 389-408

[10] S. Gudmundsson, M. Svensson On the existence of harmonic morphisms from threedimensional Lie groups, preprint, 2010.

[11] E. Heintze, On Homogeneous Manifolds of Negative Curvature, Math. Ann. 211 (1974), 23-34.

[12] T. Ishihara, A mapping of Riemannian manifolds which preserves harmonic functions, J. Math. Kyoto Univ. 19 (1979), 215-229.

[13] P. Pansu, Métriques de Carnot-Carathéodory et quasiisométries des espaces symétriques de rang un, Ann. of Math. 129 (1989), 1-60.

[14] V. Svensson, Curvatures of Lie groups, Bachelor's thesis, Lund University (2009), http://www.matematik.lu.se/matematiklu/personal/sigma/students/index.html

[15] J. Wolf, Homogenity and bounded isometries in manifolds of negative curvature, Illinois J. Math. 8 (1964), 14-18.

Department of Mathematics, Faculty of Science, Lund University, Box 118, S-221 00 Lund, SWEDEN

E-mail address: Sigmundur.Gudmundsson@math.lu.se

Department of Mathematics, Faculty of Science, Lund University, Box 118, S-221 00 Lund, SwEDEN

E-mail address: JonasCWNordstrom@gmail.com 